\title{
Improved two-temperature modeling of ultrafast thermal and optical phenomena in continuous and nanostructured metal films
}

\author{
P. Bresson,,${ }^{1,2,3}$ J-F. Bryche $\odot,{ }^{1,2, *}$ M. Besbes, ${ }^{3}$ J. Moreau, ${ }^{3}$ P-L. Karsenti, ${ }^{4}$ P. G. Charette $\odot,{ }^{1,2}$ D. Morris $\odot,{ }^{1,2,4}$ and M. Canva ${ }^{1,2}$ \\ ${ }^{1}$ Laboratoire Nanotechnologies Nanosystèmes (LN2) - CNRS UMI-3463, Institut Interdisciplinaire d'Innovation Technologique (3IT), \\ Université de Sherbrooke, 3000 Boulevard Université, Sherbrooke, J1K OA5 Québec, Canada \\ ${ }^{2}$ Institut Interdisciplinaire d'Innovation Technologique (3IT), Université de Sherbrooke, 3000 Boulevard Université, Sherbrooke, \\ J1K OA5 Québec, Canada \\ ${ }^{3}$ Université Paris-Saclay, Institut d'Optique Graduate School, CNRS, Laboratoire Charles Fabry, 91127 Palaiseau, France \\ ${ }^{4}$ Faculté des Sciences - Université de Sherbrooke - Sherbrooke, Canada
}

(Received 21 January 2020; revised 3 September 2020; accepted 8 September 2020; published 19 October 2020)

\begin{abstract}
In this work, a pump-probe experiment is used to study the ultrafast dynamics of heat transfer in thin gold films and gold nanostructures on glass substrates, following local heating by ultrashort laser pulses. Full spectrotemporal differential reflectivity and transmission maps were obtained for different film thicknesses $(30,50,80,150$, and $200 \mathrm{~nm})$ and different laser fluences $\left(0.38\right.$ to $\left.9.5 \mathrm{Jm}^{-2}\right)$. For arrays of gold nanorods, the two orthogonal probe polarizations were also acquired. We propose an improved model for these phenomena based on a modified two-temperature model that integrates thermal conduction and the three-dimensional finite element method model to link the spatiotemporal temperature maps to the spectrotemporal optical response maps. The impact of an underlying titanium adhesion layer is reported. Excellent agreement between numerical and experimental data for both the gold films and the nanostructures is shown.
\end{abstract}

DOI: 10.1103/PhysRevB.102.155127

\section{INTRODUCTION}

Thermoplasmonics [1-3] is an emerging branch of plasmonics, exploiting thermal effects in metallic nanoparticles. Long regarded as problematic, Joule effect losses due to the absorption of light by metallic nanoparticles are now considered as a potentially useful property that can be exploited in many applications. Indeed, medicine [4], magnetic recording [5], chemical catalysis [6], thermotronics [7,8], and energy conversion [9] are several areas where thermal nanosources have shown great promise. In a regime where multiphotonic ionization is negligible, the absorption of ultrashort light pulses by a metallic nanoparticle or a film leads first to the production of an out-of-equilibrium distribution of hot electrons that thermalizes within a few hundred femtoseconds (fs). At this timescale, the electronic temperature can easily reach thousands of degrees Kelvin $(\mathrm{K})$, while the lattice temperature remains unchanged. Thereafter, collisions between electrons and phonons transmits the absorbed energy to the lattice until equilibrium is reached in the illuminated area. The excess energy is dissipated by conduction in the metal and the environment. At short time scales, the heated volume is confined to the nanoparticle itself which allows precise control of thermal effects on scale far below the diffraction limit.

The first model describing these phenomena was proposed by Anisimov [10] in an attempt to explain the thermionic emission from a metal film excited by ultrashort laser pulses.

*jean-francois.bryche@usherbrooke.ca
In this paper, the authors laid the foundation for the model now more commonly known as the "Two Temperature Model". This model was used in several studies to describe the energy exchange between electron and phonon populations in thin metal films [11,12], semiconductors [11-13] or to study thermal effects in nanostructures [14]. Additions have been made to this model to account for the temperature dependence of parameters such as electron conductivity $[11,15]$ and electron density $[11,12]$, nonthermal distribution of hot electrons [16-18], and electrons thermalization [19]. However, these more complex models $[13,17,20]$ still fall short of modeling measurements over a wide timescale with high accuracy. Here we report an improved model that addresses this need. The model provides very good agreement with experimental data across a range of laser fluences, metal film thicknesses, and metal film nanostructures, over a time scale of three orders of magnitude [from hundreds of fs to a few nanoseconds (ns)].

Many techniques have been proposed to measure these ultrafast thermal effects, mainly photoemission spectroscopy [21] and pump-probe techniques [19,22,23]. We have chosen the latest to acquire spectrotemporal reflectivity and transmissivity maps following the ultrafast heating of the metallic film. The diagram of a typical experiment is shown in Fig. 1. The wavelength of the probe and the pump beams can be adjusted over the whole visible and near infrared spectra. In parallel, we developed a numerical approach to extract the spatiotemporal distribution of temperature within the sample as well as the values of the various physical parameters of the model. This approach was tested for thin gold films of different thicknesses on glass substrates in order to take material properties and volume effects into account. The measurements 

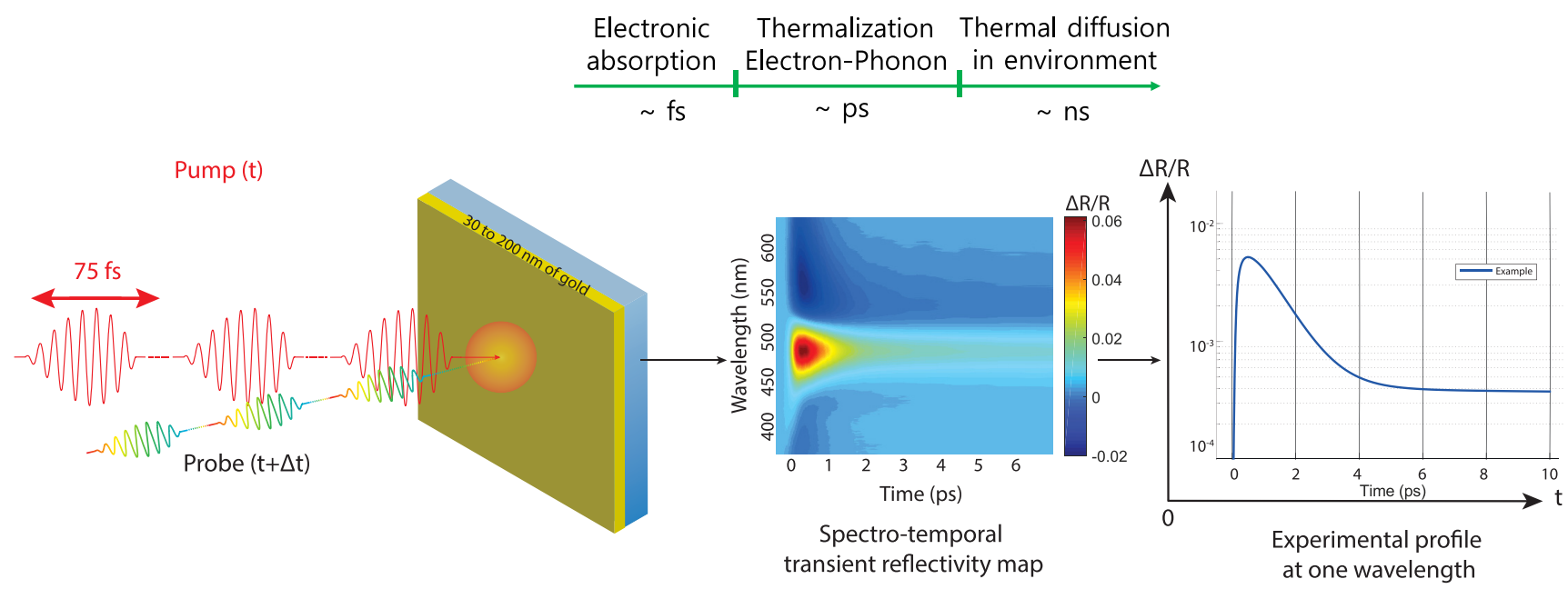

FIG. 1. Pump-probe experiment used to measure the spectrotemporal reflectivity maps of different gold samples. From these maps, timeresolved differential reflectivity profiles at a specific probing wavelength can be extracted and compared with the numerical data obtained from the model.

were done at different pump fluences (in the linear regime) to confirm the robustness of the model. Finally, we demonstrate that the model can also be applied to a sample made of an array of gold nanorods on glass substrate.

\section{MATERIALS AND METHODS}

The experimental setup is shown in Fig. 2. It is used to acquire the spectrotemporal optical response (reflectivity and/or transmission spectra) of a sample with a high temporal resolution (75 fs). The laser chain used for this experiment includes a Ti:sapphire oscillator with a regenerative amplifier (Soltice, Spectra-Physics) coupled to an optical parametric amplifier (OPA 800CF, Spectra-Physics). The OPA's output pump beam can cover a wide wavelength range, from $300 \mathrm{~nm}$ to $3 \mu \mathrm{m}$, owing to various nonlinear crystal stages. For this work, the wavelength of the pump pulses was centered at $400 \mathrm{~nm}$ to excite electrons from the gold $5 \mathrm{~d}$ band up to the conduction band above the Fermi level. The probe beam consists of a white light continuum produced by focusing the residual OPA output beam at $795 \mathrm{~nm}$ into a continuously translated 2-mm CaF2 or Saphir window (depending on the sample). The probe beam incidence angle on the sample is $\sim 5^{\circ}$ from normal incidence with a numerical aperture of $\sim 0.065$. The temporal resolution of the optical setup was determined by measuring the transient two-photon absorption signal on a $100-\mu \mathrm{m}$ thick BK7 window under the same conditions before each experiment. The resulting transient map allowed us to retrieve the overall instrument response function (IRF) and the probe's chirp. A numerical chirp correction is then applied to the data. Finally, the pump duration was measured equal to $75 \mathrm{fs}$, giving a pump probe convolution time of 92 fs. A motorized linear translation stage precisely controls the delay between the pump and probe pulses over an interval of $8 \mathrm{~ns}$, with a resolution of 6.6 fs. A noncollinear beam configuration is used to generate a linearly polarized probe beam. The pump and

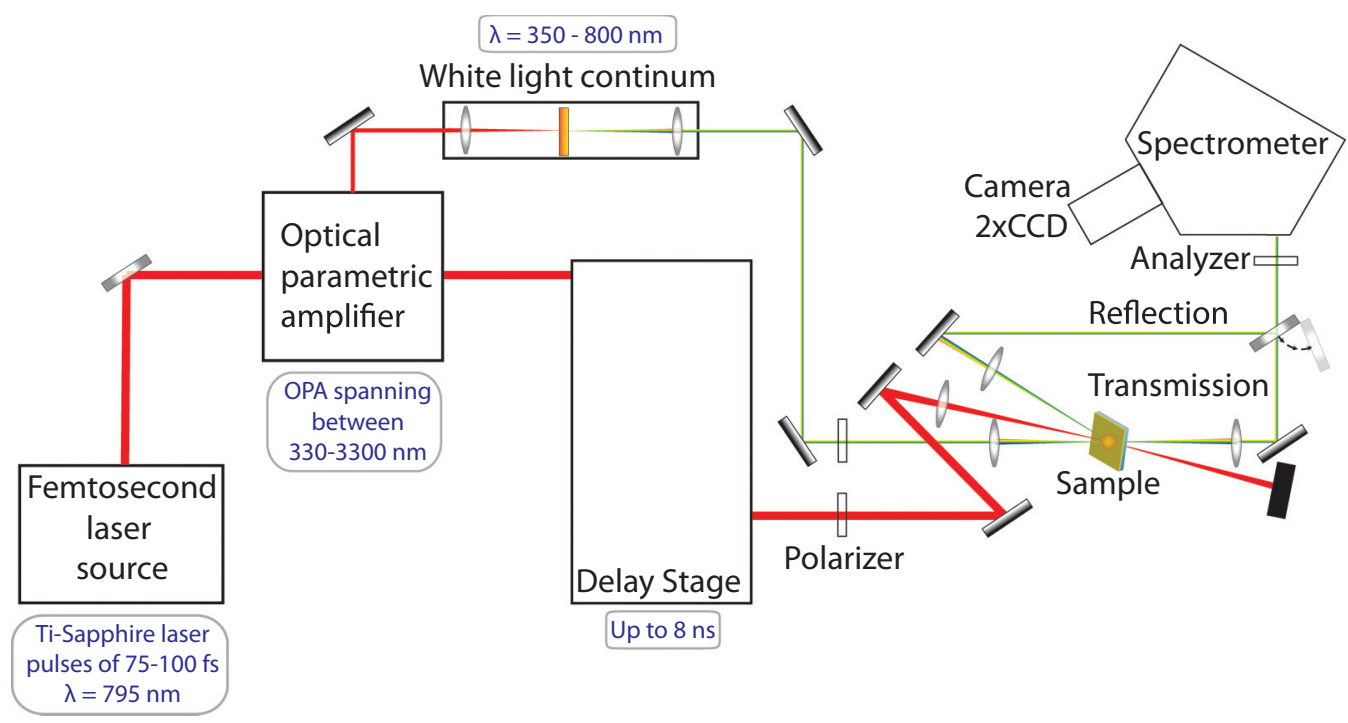

FIG. 2. Schematic of the experimental pump-probe setup for measurement of transient transmission and reflection spectrum of the sample. 


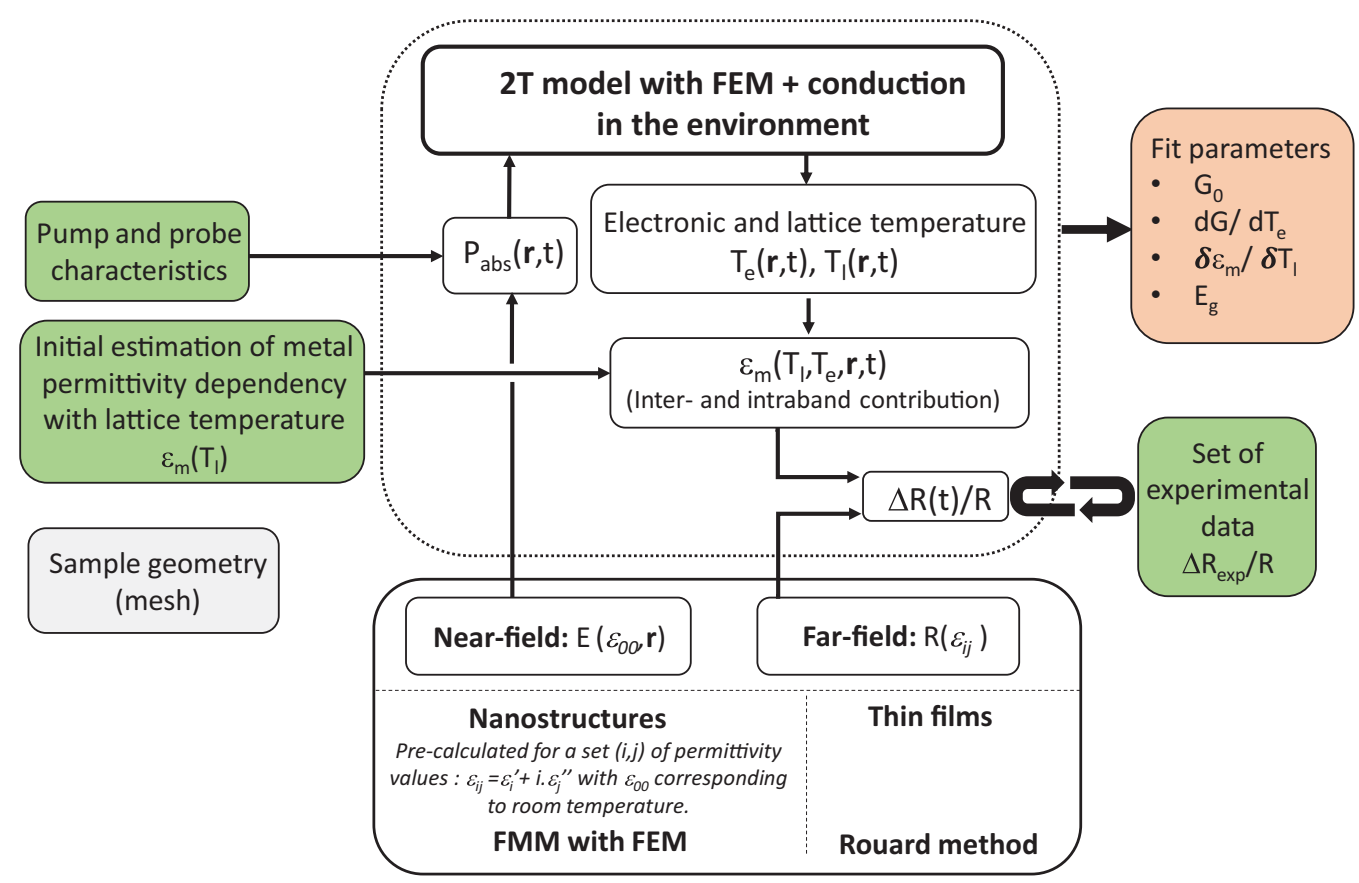

FIG. 3. Diagram of the different steps in the algorithm used to fit the model to a set of differential reflectivity values $(\Delta R(t) / R)$ measured at different pump-probe delays and pump fluences. The geometry and composition of the sample are described by a 2D multilayer or 3D FEM. The algorithm uses the two temperatures model parametrized for the optical pump conditions and sample physical parameters used in the experiments to estimate the electronic and lattice temperature spatial distributions. These in turn enable the estimation of the metal permittivity distribution, which is used to calculate the sample reflectivity and absorption coefficients.

probe beams are focused on the sample with an elliptic beam profile. Elliptic waist sizes are 160 and $70 \mu \mathrm{m}$ for the pump and 47 and $70 \mu \mathrm{m}$ for the probe. The range of pump fluences was determined experimentally to remain below the damage threshold of the gold samples $\left(75 \mathrm{~J} \mathrm{~m}^{-2}\right)$. The reflectivity and transmission spectra were acquired for the two orthogonal linear polarizations from an analyzer placed in front of the inlet slit of the spectrometer equipped with two chargecoupled device (CCD) cameras (2xS7030-1006, Spectronic Devices Ltd).

The samples consisted of thin metallic films deposited on 900- $\mu$ m-thick borosilicate BK7 D263 glass substrates. The glass substrates were first cleaned using a soaking sequence in different solutions: acetone, propan-2-ol, deionized water followed by a piranha solution (3:1). Gold films of different thicknesses $(30,50,80,100,150$, and $200 \mathrm{~nm})$ were deposited by evaporation following deposition of a 2-nm-thick titanium adhesive layer on the glass substrates. A calibrated quartz crystal was used to estimate the thickness of the deposited layers to an accuracy of $2 \mathrm{~nm}$. Actual metal film thicknesses were verified by ellipsometry. The permittivity of gold films was measured for a 150-nm-thick film deposited on a glass substrate by ellipsometry and is used in our model as a reference. The relative error on the permittivity value (limited by the stability of the light source) is estimated at $10^{-3}$ between 350 and $550 \mathrm{~nm}$ and $10^{-2}$ between 550 and $850 \mathrm{~nm}$. The nanostructured samples (see Sec. V) were fabricated using e-beam lithography: an 80-nm-thick layer of e-beam resist PMMA (polymethylmethacrylate A2) was spin-coated on the cleaned BK7 substrates, the resist was baked at $120{ }^{\circ} \mathrm{C}$ for $5 \mathrm{~min}$, followed by exposure to a $20-\mathrm{kV}$ electron beam.
The PMMA resist was developed using a solution of 9:1 propan-2-ol/water solution (1 $\mathrm{min}$ ) and samples were rinsed in water (30 s) and dried. Finally, the gold nanostructures were revealed by liftoff in acetone. Final nanostructure geometry was characterized using scanning electron microscopy (SEM).

\section{NUMERICAL MODEL}

Our finite-element-based numerical model was used to reproduce the experimental modification of the reflectivity and transmission spectra induced by local heating of the gold films. Figure 3 shows a diagram summarizing the different steps of the fitting algorithm. The key element in our approach is the use of an appropriate electromagnetic model to predict the far-field spatiotemporal relative reflectivity spectrum $\frac{\Delta R}{R}(r, t)$ from the sample, for a given wavelength and permittivity distribution $\varepsilon(r, t)$ in the metal, as well as the near-field distribution $E_{\mathrm{loc}}(r)$ for a given optical excitation. At each iteration of the algorithm, the chosen fit parameters are adjusted in the two-temperature model to calculate a new estimate of the electronic and lattice temperatures. These temperature values are then used to estimate the permittivity distribution $\varepsilon(r, t)$ and the corresponding sample reflectivity at a set of positions $r$ (fixed by the mesh size) and pump-probe delays $t$ (fixed by the temporal step). These results are then compared to a set of experimental data and a trust region algorithm is used to solve this optimization problem until convergence is achieved. From the large set of physical parameters that are involved in the two-temperature and electromagnetic models, we have shown that the experimental data can be fitted to a high degree of accuracy with only a limited number of free 
parameters, namely the electron-phonon coupling constant $G(r, t)$, the metal energy gap between the $d$ band and the Fermi level and the linear evolution of the gold permittivity with lattice temperature.

\section{A. Near-field and far-field electromagnetic modeling}

To calculate the spatiotemporal distribution of the electromagnetic field inside the sample, as well as the far-field reflectivity spectrum of the probe beam, two different approaches are used depending on the geometry of the sample. For thin continuous films, the distribution of temperature inside the illuminated area depends only on $z$, the axis perpendicular to the film plane. The volume of the sample can be discretized in a series of horizontal layers and the Rouard method [24] can then be used to determine the reflectivity and transmittivity spectra from the permittivity. A value of $15 \mathrm{~nm}$ was chosen for these virtual horizontal layers, half the skin depth in gold (30 nm at $400 \mathrm{~nm}$ ). For nanostructured films with complex geometry, we use a hybrid approach combining finite elements (FEM) with the Fourier modal method [25] to calculate both the near-field and the far-field responses. This hybrid approach gives accurate results and significantly reduces the computer memory requirements compared to a pure FEM approach. Adaptative volume meshing (1 nm to tens of $\mathrm{nm}$ resolution) is used to discretize the more or less rapid variations in fields around the nanostructures and the plasmonic hot spots. With the appropriate boundary conditions, it is possible to model a single nanostructure or a periodic array of nanostructures.

In both approaches, the constitutive equation and linear optical response of a conductive material to plane-wave illumination can be used to relate the power density absorbed per unit volume, $P_{\mathrm{abs}}(r, t)$, to the local electromagnetic field, $E_{\mathrm{loc}}(r, t)$ :

$$
P_{\mathrm{abs}}(r, t)=\frac{1}{2} \varepsilon_{0} \omega\left|E_{\mathrm{loc}}(r, t)\right|^{2} \varepsilon_{m}^{\prime \prime}(r, t)
$$

The term $\varepsilon_{m}^{\prime \prime}(r, t)$ is the imaginary part of the metal permittivity and $\omega$ is the frequency of the excitation. In this calculation of the heat source term, the value of gold permittivity is taken from the literature at $300 \mathrm{~K}$ and is considered constant over the duration of the excitation pump pulse. The incident illumination profile is assumed to be Gaussian.

This hybrid method allows us to determine the far-field reflectivity and transmittivity of the sample at the probe wavelength. However, the calculation time is too long if this is performed at each iteration step of the algorithm. Instead, far-field reflectivity and transmittivity are precalculated over the metal permittivity dynamic range corresponding to the electronic and lattice temperature extrema reached during the experiments (typical relative variations of permittivity are of the order of $30 \%$ for the real part and $50 \%$ for the imaginary part). The optimization algorithm used interpolation based on this densely pre-computed table of values to estimate far-field reflectivity and transmittivity at each step.

\section{B. Two-temperature model with thermal conduction}

In our model, the heat source $S(r, t)$ is linked to the absorbed power density $P_{\mathrm{abs}}(r, t)$ in the metal film. The density of thermal energy per unit volume $N_{E}(r, t)$ absorbed from the excitation pulse is assumed to be completely transferred to the nonequilibrium distribution of hot electrons in the metal film. These nonthermalized electrons will transport energy by ballistic motion and then transfer their energy to the electron and phonon populations through electron-electron and electron-phonon scattering mechanisms [26,27]. To provide a more accurate description of the phenomena occurring during optical excitation, we have chosen not to use the approximation of an instantaneous pump excitation, used by some authors [16,17], but instead include the temporal evolution of the pump pulse via the term $P_{\mathrm{abs}}(r, t)$. The temporal variation of $N_{E}$ can therefore be written as [16-18]

$$
\begin{aligned}
S(r, t) & =\frac{d N_{E}(r, t)}{d t} \\
& =P_{\mathrm{abs}}(r, t)-\frac{N_{E}(r, t)}{\tau_{e e}}-\frac{N_{E}(r, t)}{\tau_{\mathrm{th}}}+v_{e} . \nabla N_{E}(r, t),
\end{aligned}
$$

where $\tau_{e e}$ and $\tau_{\text {th }}$, are, respectively, the nonthermalized electron-electron and electron-phonon relaxation times and and $v_{e}$ is the speed of the ballistic electrons. The following equation [27] allows us to calculate $v_{e}$ taking into account its dependence on the electronic temperature:

$$
v_{e}=\sqrt{\frac{2 \mu+3 k_{B} T_{e}}{m_{e}}},
$$

where $k_{B}$ is the Boltzmann constant and $m_{e}$ is the electron mass. The $v_{e}$ value is close to the Fermi velocity $v_{F}$ and around $1.5 \times 10^{6} \mathrm{~m} \mathrm{~s}^{-1}$ for the considered range of temperature.

The spatiotemporal behavior of the electron and lattice temperatures $\left(T_{e}\right.$ and $\left.T_{l}\right)$ of the metal as well as the temperature of the environment are described by the following coupled equations of the two-temperature model:

$$
\begin{aligned}
& C_{e}\left(T_{e}\right) \frac{d T_{e}(r, t)}{d t}= \nabla\left(k_{e}\left(T_{e}, T_{l}\right) \nabla T_{e}(r, t)\right) \\
&-G\left(T_{e}(r, t)-T_{l}(r, t)\right)+\frac{N_{E}(r, t)}{\tau_{e e}}, \\
& C_{l} \frac{d T_{l}(r, t)}{d t}= \nabla\left(k_{l} \nabla T_{l}(r, t)\right) \\
&+G\left(T_{e}(r, t)-T_{l}(r, t)\right)+\frac{N_{E}(r, t)}{\tau_{\text {th }}}, \\
& C_{\text {air,glass }} \frac{d T_{\text {air,glass }}(r, t)}{d t}=\nabla\left(k_{\text {air,glass }} \nabla T_{\text {air,glass }}(r, t)\right) .
\end{aligned}
$$

The last equation being applied both to the glass substrate and the air above the sample. The contribution of convection in the surronding air is negligible on the time scale of our experiments. The energy loss by Planck emission is also negligible considering the low intrinsic efficiency of this phenomenon (See Table S2 in the Supplemental Material [43] for a comparison of the typical heat flux for all these heat transfer 
mechanisms). Far from the sample, ambient temperature was used as a boundary condition.

The thermal capacity and conductivity of the gold lattice were considered constant within the range of electronic temperatures achieved in all our experiments as we are above the gold Debye temperature and far below Fermi temperature. Typical values found in the literature were chosen for the thermal parameters of the BK7 glass substrate, which also have negligible variations with temperature $\left(C_{l}=2.492 \times 10^{6} \mathrm{~J} \mathrm{~m}^{-3} \mathrm{~K}^{-1} \quad\right.$ and $\quad k_{l}=2.6 \mathrm{~W} \mathrm{~m}^{-1} \mathrm{~K}^{-1}$ for gold lattice, $C_{\text {glass }}=2.0194 \times 10^{6} \mathrm{~J} \mathrm{~m}^{-3} \mathrm{~K}^{-1}$ and $k_{\text {glass }}=1.05 \mathrm{~W} \mathrm{~m}^{-1} \mathrm{~K}^{-1}$ for BK7 glass and $C_{\text {air }}=$ $1.1899 \times 10^{3} \mathrm{~J} \mathrm{~m}^{-3} \mathrm{~K}^{-1}$ and $k_{\text {air }}=0.024 \mathrm{~W} \mathrm{~m}^{-1} \mathrm{~K}^{-1}$ for air). Thermal parameters for air vary significantly with temperature but were nevertheless kept constant as their impact on the overall evolution of the system is small (see Table S1 in Supplemental Material for the justification). Previous studies [28] have shown that the thermal excitation of the $d$-band electrons significantly impacts values of the electronic thermal capacity $C_{e}$, the conductivity $k_{e}$ and the electron-phonon coupling constant at high electronic temperature. For electronic temperatures below a few thousand Kelvin, a linear dependence was found to be a good estimation for the evolution of these parameters: $C_{e}=\gamma T_{e}$ with $\gamma=67.96 \mathrm{~J} \mathrm{~m}^{-3} \mathrm{~K}^{-2}$ [13], $k_{e}=318 T_{e} / T_{l} \mathrm{~W} \mathrm{~m}^{-1}$ [19] and $G=G_{0}+T_{e} d G / d T_{e}$. The value of $G_{0}$ and $d G / d T_{e}$ were chosen as free parameters in the fitting algorithm.

The electron thermalization time $\tau_{e e}$ is assumed to be inversely proportional to the square of the electronic temperature [13], while the electron-phonon thermal energy relaxation time $\tau_{\text {th }}$ can be estimated by considering a linear approximation [18] of the evolution of the system energy (with $U_{e}=\frac{\gamma T_{e}^{2}}{2}$ ):

$$
\tau_{\mathrm{th}}=\frac{U_{e}-U_{e}(\infty)}{\frac{d U_{e}}{d t}}=\frac{\gamma\left(T_{e}^{2}-T_{l}^{2}\right)}{2 \times G\left(T_{e}-T_{l}\right)}=\frac{\gamma\left(T_{e}+T_{l}\right)}{2 \times G} .
$$

Examples of spatial electronic and lattice temperatures maps are presented in sections below (see Fig. 7).

Finally, the heat flux continuity equations at the different interfaces account for the transfer of thermal energy between the film and its environment (substrate/air) (equations not shown).

\section{Permittivity and temperature}

As explained above, both the calculation of the reflectivity and the pump power absorbed per unit volume depend on the estimation of the metal permittivity at a given electronic and lattice temperatures (permittivity of the substrate is considered constant). Using a first order approximation, we have:

$$
\varepsilon\left(T_{e}, T_{l}\right)=\varepsilon_{T_{\mathrm{amb}}}+\left(\frac{\delta \varepsilon}{\delta T_{e}}\right)_{T_{l}=T_{\mathrm{amb}}} \Delta T_{e}+\left(\frac{\delta \varepsilon}{\delta T_{l}}\right)_{T_{e}=T_{\mathrm{amb}}} \Delta T_{l} .
$$

The first term is the gold permittivity at room temperature, measured by ellipsometry. The second term is the variation of permittivity with electronic temperature, which depends both on intraband transitions, described by the classical Drude model [29] as well as on interband transitions, from the flat $d$ band to the upper band states above the Fermi level:

$$
\left(\frac{\delta \varepsilon}{\delta T_{e}}\right)_{T_{l}=T_{\mathrm{amb}}}=\frac{\delta \varepsilon_{\mathrm{ib}}}{\delta T_{e}}+\frac{\delta \varepsilon_{\mathrm{drude}}}{\delta T_{e}}
$$

with

$$
\varepsilon_{\text {drude }}=\varepsilon_{\infty}-\frac{\omega_{p}^{2}}{\omega^{2}+i \frac{\omega}{\tau\left(T_{e}, T_{l}\right)}} .
$$

The characteristic scattering time $\boldsymbol{\tau}$ is known empirically and can be written as follows [28,30]:

$$
\frac{1}{\tau}=\frac{1}{\tau_{e-e}}+\frac{1}{\tau_{e-\mathrm{ph}}}+C \frac{v_{F}}{R}=A T_{e}^{2}+B T_{l}+\frac{v_{F}}{R},
$$

where $\tau_{e-e}$ and $\tau_{e-\mathrm{ph}}$ are the electron-electron and electronphonon scattering times, respectively. $\mathrm{R}$ is the characteristic size of the nanostructure (infinite for films). The constants $A=1.2 \times 10^{7} \mathrm{~K}^{-2} \mathrm{~s}^{-1}$ and $B=1.23 \times 10^{11} \mathrm{~K}^{-2} \mathrm{~s}^{-1}$, and $v_{F}=$ $0.84 \times 10^{6} \mathrm{~m} \mathrm{~s}^{-1}$ are taken from literature [28]. For thin gold films and a probe wavelength between 400 and $600 \mathrm{~nm}$, this contribution to the permittivity is at least one order of magnitude lower compared to the interband transition for the imaginary part of the permittivity and three orders of magnitude lower for the real part of the permittivity.

For the interband transition, to accurately describe the spectral features in the optical response, the change in permittivity has to be related to the change of the electron distribution function with a model developed by Rosei $e t$ al. [30-33] were the L band structure of gold is considered [34]:

$$
\frac{\delta \varepsilon^{\prime \prime} \mathrm{ib}}{\delta T_{e}}=\frac{C^{3}}{(\hbar \omega)^{2}} \int_{E_{\min }}^{E_{\max }} D(E, h \omega)\left(-\frac{\partial f(E)}{\partial T_{e}}\right) d E,
$$

where $C$ is an experimental proportionality factor which, for gold films, was experimentally found to be independent of pump fluence and film thickness with a value equal to $2.12 \times 10^{-9} \mathrm{eV} \mathrm{m}$, where $f$ is the Fermi distribution function, and $D$ is the energy distribution of the joint density of states [see Supplemental Material for the expression of $D$ in gold as well band structures (Figs. S1-3)]. Non thermalized electrons have a negligible impact on the permittivity value. From the variation of the imaginary part of the permittivity, the Kramers-Kronig equations [35] can be used to calculate the variation of the real part of the interband permittivity $\varepsilon^{\prime}{ }_{\mathrm{ib}}$ :

$$
\frac{\delta \varepsilon_{\mathrm{ib}}^{\prime}}{\delta T_{e}}(\omega)=\frac{1}{\pi} P \int_{-\infty}^{\infty} \frac{\delta \varepsilon^{\prime \prime} \mathrm{ib}}{\delta T_{e}}\left(\omega^{\prime}\right) \frac{1}{\omega^{\prime}-\omega} d \omega^{\prime},
$$

where $P$ is the Cauchy principal value required that defines the integral around the singularity $\omega$. Convergence is always achieved as permittivity must go to zero at infinity. Combining these equations gives the wavelength dependence of the real and imaginary parts of permittivity of the metallic film [Figs. 4(a) and 4(b)], for different electronic temperatures.

The variation of the metal permittivity as a function of the lattice temperature in Eq. (8) is much more difficult to estimate. Several models exist in the literature to describe the permittivity of metals and thin films [29,36,37], but none of these models are universal as the thickness, surface roughness, and deposition process have a major influence on its optical properties. Experimental measurement of temperature and wavelength dependence of optical properties for 

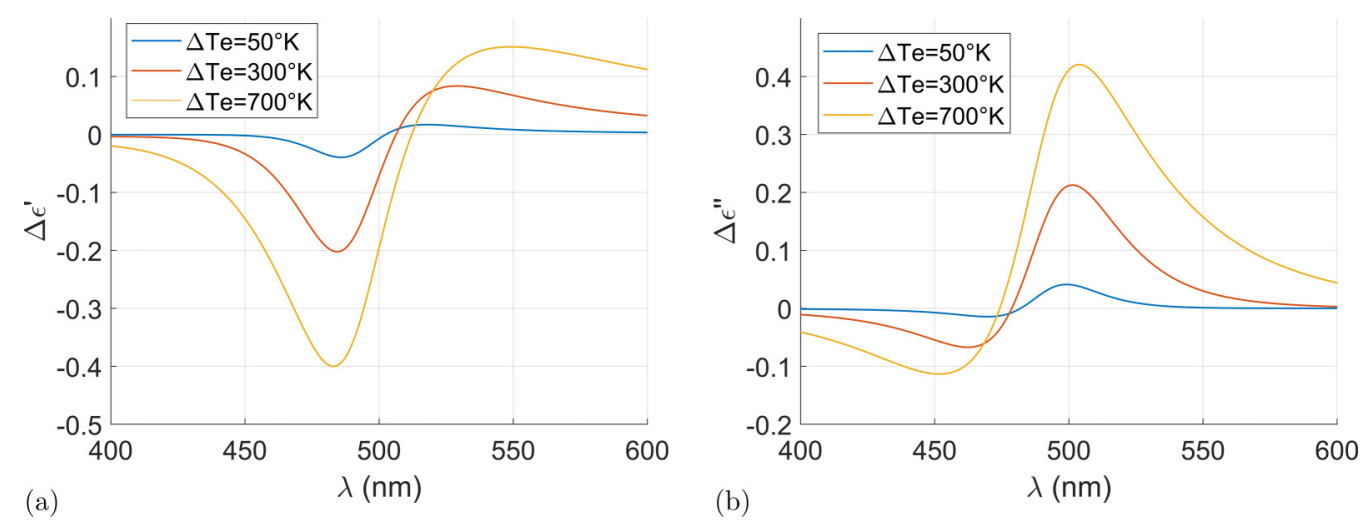

FIG. 4. (a) Variation of the real part of the permiittivity, (b) Variation of the imaginary part of the permittivity, considering intrad- and interband transition for different electronic temperature.

thin metallic films can be successfully fitted with extended Drude-Lorentz models [38], but the number of free parameters is too large to make any prediction. For this study, to address this problem, we have chosen to directly measure by ellipsometry the permittivity of a 150-nm-gold film between 380 and $890 \mathrm{~nm}$ (Fig. S6 in the Supplemental Material) and between 20 and $110{ }^{\circ} \mathrm{C}$. The real part of the optical index was found to be quasiconstant $(<1 \%)$ within this range of
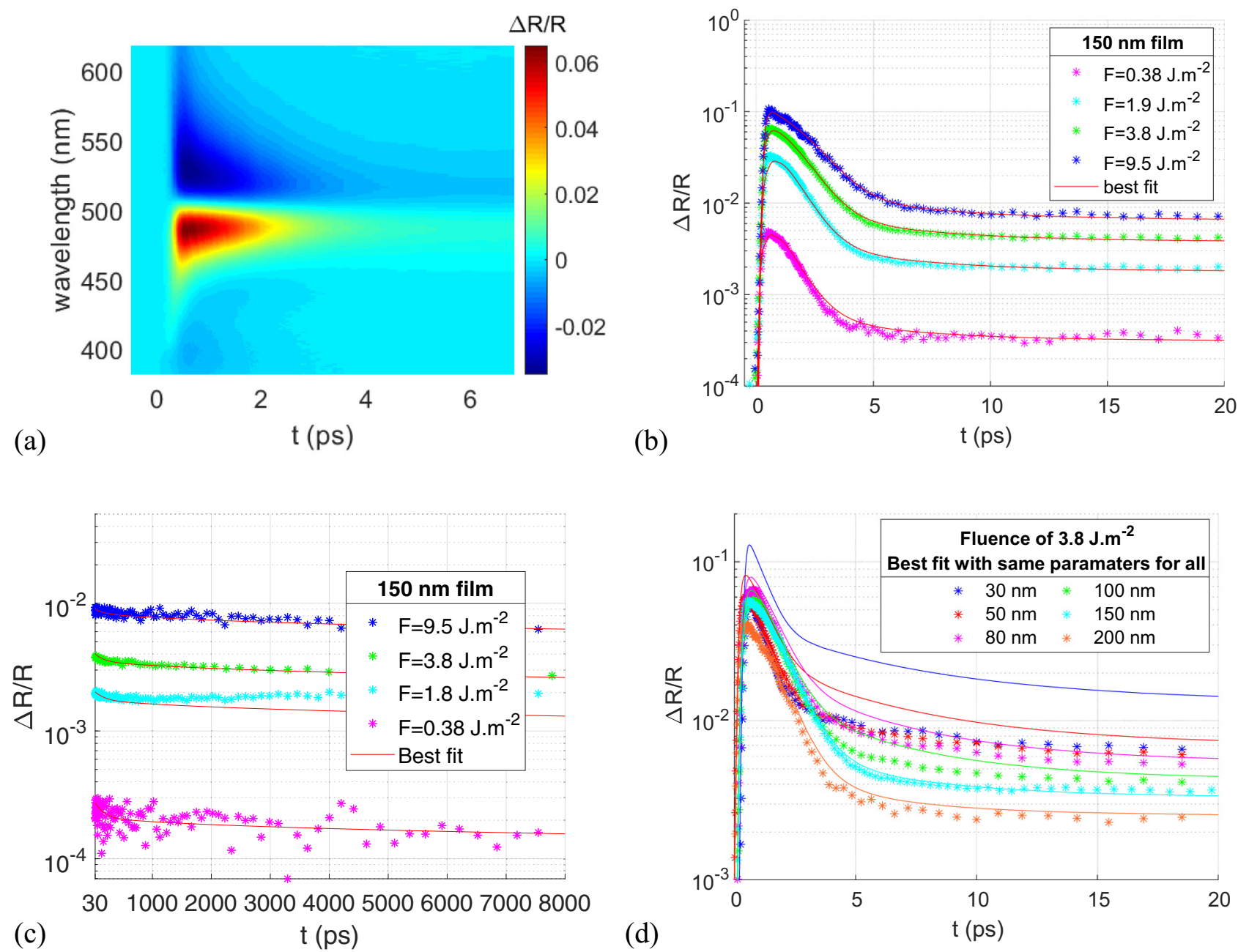

FIG. 5. (a) Example of spectrotemporal relative reflectivity map acquired from a 150-nm-gold film with a pump at $400 \mathrm{~nm}$. (b) Temporal evolution extracted from spectrotemporal maps at the maximum of variation $(\sim 490 \mathrm{~nm})$, acquired at different pump fluences and best fit for a $150 \mathrm{~nm}$ gold film. (c) Best fit at long time scale (up to $8 \mathrm{~ns}$ ) for a 150-nm-gold film at different pump fluences. (d) Temporal evolution for different gold film thicknesses and best fit for a fluence of $3.8 \mathrm{~J} \mathrm{~m}^{-2}$ with the same parameters for all. 
temperatures, while the imaginary part shows a linear dependence with temperature. From these measurements, the value and evolution with temperature of the gold permittivity complex value can be estimated. At $486 \mathrm{~nm}$, we found: $d \varepsilon / d T=$ $-2.4 \times 10^{-3}(1-i) \mathrm{K}^{-1} \pm(-0.5+0.6 i) \times 10^{-3} \mathrm{~K}^{-1}$. This quantity is used as the second term of Eq. (10), even if it is not rigorously the case since for this ellipsometry measurement, the electronic temperature was equal to the lattice temperature.

Variation of the permittivity of the glass and air environments with temperature was found to have negligible effect on the overall reflectivity of the sample (relative variation of reflectivity of $10^{-6} \mathrm{~K}^{-1}$ for air and $10^{-8} \mathrm{~K}^{-1}$ for glass). Finally, due to the difficulty of measuring the temperature dependency of the permittivity of the titanium adhesion layer under the gold film, due to its very small thickness, it was kept constant in the model. The impact of this adhesion layer on the thermoplasmonics response is further described below (See Figs. 8 and S5).

\section{EXPERIMENTAL AND NUMERICAL RESULTS ON THIN GOLD FILMS}

We compare numerical modeling results with experimental data measured from gold films of six different thicknesses $\left(h_{\text {gold }}: 30,5080,100,150,200 \mathrm{~nm}\right)$ and under four different pump fluences ( $\Phi_{0}$ ranging from 0.36 to $9.5 \mathrm{~J} \mathrm{~m}^{-2}$ ). The time-series of maximum relative spectral reflectivity were calculated from the spectrotemporal maps acquired with each of the 24 experimental conditions above using our pumpprobe setup, yielding a three-dimensional (3D) data cube $\max (\Delta R / R)\left(t, h_{\text {gold }}, \Phi_{0}\right)$ for use in model fitting. The energy per excitation pulse incident on the sample was estimated by measuring the pump beam power (stability: $\sim 10 \%$ for power $\leqslant 50 \mathrm{~mW}, \sim 1 \%$ for power $>50 \mathrm{~mW}$ ) and beam waist (accuracy: $\pm 5 \mu \mathrm{m}$ ).

Figure 5(a) shows an example of such a spectrotemporal map for a 150-nm-gold film at a pump fluence of $3.8 \mathrm{~J} \mathrm{~m}^{-2}$ (corresponding to a pulse energy of $100 \mathrm{~nJ}$ ). The pump wavelength was set to $400 \mathrm{~nm}$ and the probe spectral windows range from 350 to $650 \mathrm{~nm}$ for all samples. Absorption of the pump energy leads to a rapid increase in the electronic temperature which induces a modification of the gold permittivity amplitude and spectrum. As we measure the relative variation of reflectivity of the sample, Fig. 5(a) can be directly related to this variation of permittivity which was shown in Fig. 4(b). The decrease in the imaginary part of the gold permittivity spectrum in the blue part of the spectrum is responsible for the positive $\Delta R / R$ peak in Fig. 5(a) around 475 $\mathrm{nm}$, as it corresponds to a transient lower absorption of the sample, whereas the negative $\Delta R / R$ peak around $525 \mathrm{~nm}$ in Fig. 5(a) corresponds to a transient higher absorption related to the increase in the imaginary part of the permittivity. From these maps, temporal profiles are extracted at the wavelength where the reflectivity variation in wavelength is maximum $(\sim 490 \mathrm{~nm})$. Figure 5(b) shows a set of experimental temporal profiles for a film of $150 \mathrm{~nm}$ at all fluences. Higher fluences correspond to higher electronic temperatures $T_{e}$ and lattice temperatures $T_{l}$ which induce larger modifications of the gold permittivity and therefore reflectivity. The different time scales are also apparent: at first $(<1 \mathrm{ps})$, the absorption of the pump pulse results in the reflectivity variation reaching a maximum, then (within a few ps) we observe a fast decrease corresponding to the energy exchange between electrons and phonons until an equilibrium is reached. Finally, conduction in the glass substrate and surrounding air returns the system to its initial state (after a few ns).

To compare these experimental data with the numerical results, the subset of data $\Delta R / R\left(t, h_{\mathrm{gold}}=150 \mathrm{~nm}, \Phi_{0}\right)$ was fitted using the algorithm described above and with a minimum number of free parameters: only the electron-phonon coupling constant and its dependency on electronic temperature. The same set of parameters is used for all fluences. Fitted curves are in excellent agreement with the experimental data for all fluences with a root mean square error (rmse) of 0.05 for the curves normalized by their maxima [Fig. 5(b)]. The best fitting curves were obtained with the following value of $G_{0}=2.27 \pm 0.06 \times 10^{16} \mathrm{~W} \mathrm{~m}^{-3} \mathrm{~K}^{-1}$, in agreement with reported values measured or predicted in the literature [39]. The first order dependence of $G_{0}$ on electronic temperature was found to be close to zero as the maximum $T_{e}$ reached is not high enough for this correction to have a significant impact on the fit $\left(d G / d T_{e}=10^{-4}\left[0 ; 4 \times 10^{-4}\right] 10^{13} \mathrm{~W} \mathrm{~m}^{-3} \mathrm{~K}^{-2}\right)$. Finally, as the model includes conduction losses, reflectivity variations were also fitted at longer time scales for all samples, albeit with a lower time resolution, as shown in Fig. 5(c). With the same parameters used previously, the experimental data can be fitted for all fluences up to $8 \mathrm{~ns}$. Thus, this model can quantitatively extract the temporal evolution of the electronic and lattice temperatures from the reflectivity variations in 150-nm-thin gold films, over a large range of pump fluences.

The experimental set of data $\Delta R / R\left(t, h_{\mathrm{gold}}, \Phi_{0}=3.8 \mathrm{~J} \mathrm{~m}^{-2}\right)$ obtained for different gold film thicknesses, at a fixed fluence of $3.8 \mathrm{~J} \mathrm{~m}^{-2}$, is shown in Fig. $5(\mathrm{~d})$. On the first few ps, the $\Delta R(t) / R$ curves are very similar, which is an indication that the film heating phenomenon depends only slightly on its thickness. For the 200-nm-thickness, we start to observe a slightly lower maximum as only the upper part of the film is heated by the pump, reducing the overall reflectivity change of the sample. Conversely, there is a significant difference between the different curves at longer pump-probe delays. As expected, the lattice temperature increases as the thickness of the film decreases since a smaller volume of metal absorbs the energy. The solid lines correspond to the best fit of the data set using the same values of $G_{0}$ and $d G / d T$. Values of gold permittivity and lattice temperature dependence, measured for the 150-nm sample, were also used for all other gold film thicknesses. As can be seen on Fig. 5(d), a significant deviation between the fitted curves and the experimental data is observed (with an rmse of 0.4 for the $30 \mathrm{~nm}$ data) which indicates that the parameters of the model cannot be considered constant as the film thickness changes.

Consequently, a better agreement is obtained by allowing the parameters $G_{0}, d G / d T_{e}$, and $|\delta \varepsilon / \delta \mathrm{T}|$ to vary with gold thickness (but not with fluence). Figure 6 shows an example of the best fit obtained for a fluence of $\Phi_{0}=3.8 \mathrm{~J} \mathrm{~m}^{-2}$. Table I shows the model parameters for all film thicknesses and fluences, with the confidence interval. 


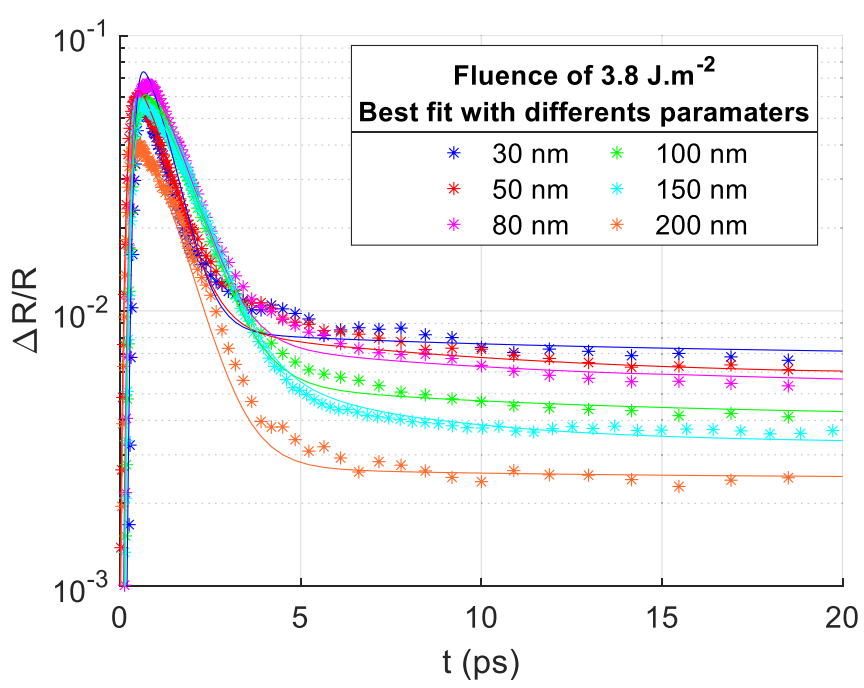

FIG. 6. Example of temporal evolution extracted from spectrotemporal maps of the maximum variation $(\lambda=\sim 490 \mathrm{~nm})$ for different gold film thicknesses at a laser fluence of $3.8 \mathrm{~J} \mathrm{~m}^{-2}$. The solid lines correspond to the best fitting curves.

$G_{0}$ is slightly dependent on the gold film thickness with a tendency to increase at lower thicknesses. The first order temperature correction to $G_{0}, d G / d T_{e}$ is also more and more significant as the thickness of the film is reduced and the maximum electronic temperature reached a higher value at peak absorption. Finally, the variation of gold permittivity with temperature is identical, within the error bars, for all films. The values of $G o$ and $d G / d T_{e}$ for the 30-nm-gold film, which are higher than for the other thicknesses, as well the higher rmse (0.14), may reveal the effects of interfaces for thin films response and the need for more precise values of thickness, adhesion layer, and roughness.

The curve fitting algorithm allows us to determine the spatiotemporal evolution of the electronic and lattice temperatures and thus to better understand the anisotropic heat conduction mechanisms inside the sample. Figure 7 shows this evolution profile for a 200-nm-gold film surrounded by air $(z>200 \mathrm{~nm})$ and the glass substrate $(z<0 \mathrm{~nm})$. For a low pump-probe delay of $100 \mathrm{fs}$, the spatial distribution of the electronic temperature in the gold film is strongly correlated to the absorption profile in this layer. It is noted that the electron temperature reaches a maximum value $\left(500^{\circ} \mathrm{C}\right)$, directly at the center of the pump beam spot at the top surface of the gold film [Fig. 7(a)]. At this short delay, the lattice temperature inside the gold film remains mostly unchanged as the electronphonon relaxation mechanisms are much slower [Fig. 7(b)]. After a delay of $500 \mathrm{fs}$, the ballistic electrons and the fast electronic conduction mechanisms lead to a large extension of the electronic temperature inside the gold film [Fig. 7(c)]. There is also a slight heat transfer to the air medium and to the glass substrate [Fig. 7(d)] as the temperature of the lattice start to increase on the top surface of the gold film but also, interestingly, in the underlying titanium adhesion layer. Finally, after $20 \mathrm{ps,} \mathrm{the} \mathrm{electronic} \mathrm{temperature} \mathrm{becomes}$ homogeneous inside the film, while the lattice temperature distribution still shows higher values at the interfaces with significant heat transfer in the air [Figs. 7(e) and 7(f)]. It can also be seen that the conduction inside the plane of the sample is negligible (along the $\boldsymbol{r}$ axis). Examples of temperature maps are also provided for a thinner film $(50 \mathrm{~nm})$ in the Supplemental Material (Fig. S4).

As specified above, all these simulations include the titanium adhesion layer present between the gold film and the substrate, which is almost mandatory from an experimental point of view to ensure a good adhesion of the gold film. Yet, even if this adhesive layer is only a few nanometers thick, it significantly impacts the thermoplasmonics response of the sample. To illustrate this point, we have fabricated a 50-nmgold film without any adhesion layer. Figure 8 shows the experimental reflectivity variation profiles with and without the titanium layer for a pump fluence of $3.8 \mathrm{~J} \mathrm{~m}^{-2}$. Without the adhesion layer, the amplitude of $\Delta R / R$ and therefore the maximum electronic temperature reached is increased by almost $40 \%$, and the decay time is also significantly longer. In this case, the curve fitting procedure does not perfectly reproduce the experimental data (fitted curve not shown). This indicates a significant modification of the thermal properties

TABLE I. Mean value of the fitted parameter with estimated uncertainty for the different film thicknesses. For each gold film, the whole set of experimental curves measured at different pump fluences were fitted using the same parameter values.

\begin{tabular}{|c|c|c|c|c|}
\hline & $G_{0}\left(10^{16} \mathrm{~W} \mathrm{~m}^{-3} \mathrm{~K}^{-1}\right)$ & $d G / d T_{e}\left(10^{13} \mathrm{~W} \mathrm{~m}^{-3} \mathrm{~K}^{-2}\right)$ & $|\delta \varepsilon / \delta T| /|\delta \varepsilon / \delta T|_{150 \mathrm{~nm}}$ & RMSE \\
\hline $30 \mathrm{~nm}$ & $\begin{array}{c}3.24 \\
{[2.43 ; 4.04]}\end{array}$ & $\begin{array}{c}2.9 \\
{[2.0 ; 3.8]}\end{array}$ & $\begin{array}{c}0.82 \\
{[0.62 ; 1.02]}\end{array}$ & 0.14 \\
\hline $80 \mathrm{~nm}$ & $\begin{array}{c}2,45 \\
{[2.41 ; 2.44]}\end{array}$ & $\begin{array}{c}1.1 \\
{[1.0 ; 1.2]}\end{array}$ & $\begin{array}{c}1.08 \\
{[1.07 ; 1.09]}\end{array}$ & 0.036 \\
\hline $150 \mathrm{~nm}$ & $\begin{array}{c}2.26 \\
{[2.19 ; 2.32]}\end{array}$ & $\begin{array}{c}0.1 \\
{[0 ; 0.3]}\end{array}$ & $\begin{array}{c}0.92 \\
{[0.81 ; 1.03]}\end{array}$ & 0.045 \\
\hline $200 \mathrm{~nm}$ & $\begin{array}{c}2.7 \\
{[2.57 ; 2.83]}\end{array}$ & $\begin{array}{c}0 \\
{[0 ; 0.4]}\end{array}$ & $\begin{array}{c}0.98 \\
{[0.82 ; 1.14]}\end{array}$ & 0.07 \\
\hline
\end{tabular}



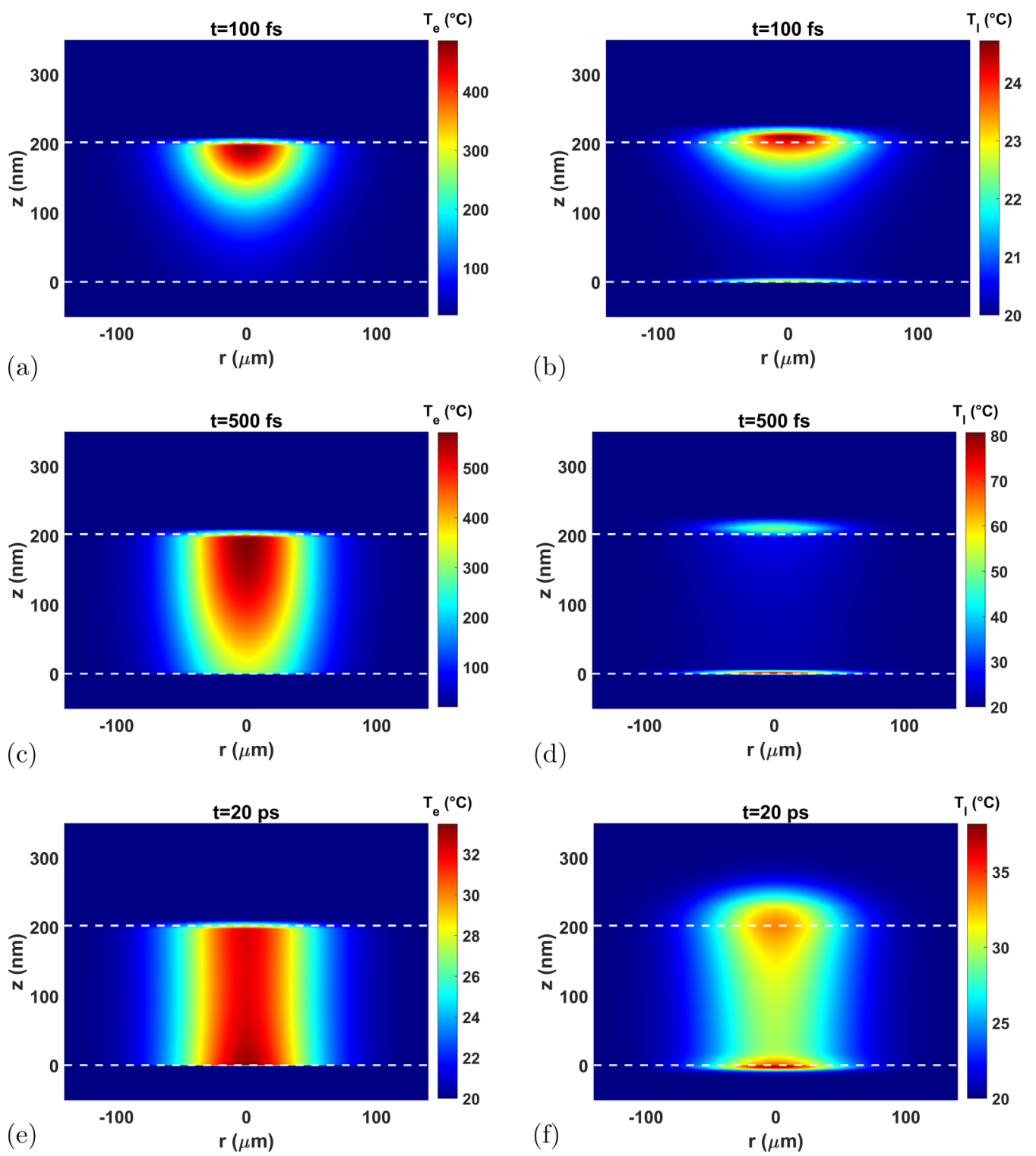

FIG. 7. Spatial distribution ( $x z$ plane) of the electronic (a),(c),(e) and lattice temperatures (b),(d),(f) in a 200-nm thin film at 3 different time delays between the pump and probe beams (100 fs, $500 \mathrm{fs}$ and $20 \mathrm{ps})$. The film interfaces are marked as dashed lines at $z=0$ and $z=200 \mathrm{~nm}$.

of the gold film, most probably due to a complex and porous interface between the glass substrate and the gold film when no adhesion layer is present.

\section{RESULTS ON NANORODS ARRAYS}

We also verified our model on experimental results obtained for a metallic nanorods array. The pump-probe measurements were done in transmission since the signal-tonoise ratio is much higher for such geometry. We used gold nanorods (theoretical width $l=40 \mathrm{~nm}$, length $L=80 \mathrm{~nm}$, and height $h=30 \mathrm{~nm})$ in an asymmetric array $\left(P_{x}=130 \mathrm{~nm}\right.$ and $P_{y}=300 \mathrm{~nm}$ ) on a BK7 substrate, as schematically shown in Fig. 9(a). An adhesion layer of titanium (2-nm thickness) was used between the gold and BK7. This titanium layer is thin enough that it does not shift or suppress plasmonic resonances [40]. The actual shape of the nanorods measured by SEM were implemented in the FEM model of the substrate geometry: the nanorods were modeled with round corners and a size of $52 \times 80 \times 30 \mathrm{~nm}$ keeping the same volume as the theoretical nanorod. The positions of plasmonic resonances $(525$ and $630 \mathrm{~nm}$ ) for the two incident polarizations are shown in Fig. 9(b). The pump wavelength was centered at $400 \mathrm{~nm}$. The pump and probe beams are linearly polarized, with the pump beam polarization oriented along the long axis of the rods and the probe beam polarization axis fixed at an angle of $45^{\circ}$ from the pump [see Fig. 9(a)]. In detection, an analyzer allows to measure the two orthogonal linear polarization components of the transmitted probe beam $\left(P_{\|}\right.$and $\left.P \perp\right)$. We observe from the relative transmission maps in Figs. 9(c) and 9 (d) that the maximum variation of the $\Delta T / T$ signal is at $\sim 620 \mathrm{~nm}$ for the polarization parallel to the rod and $\sim 545$ $\mathrm{nm}$ for the orthogonal polarization. The position of the latter maximum is close to the interband transition $\mathrm{L}$ of gold which 


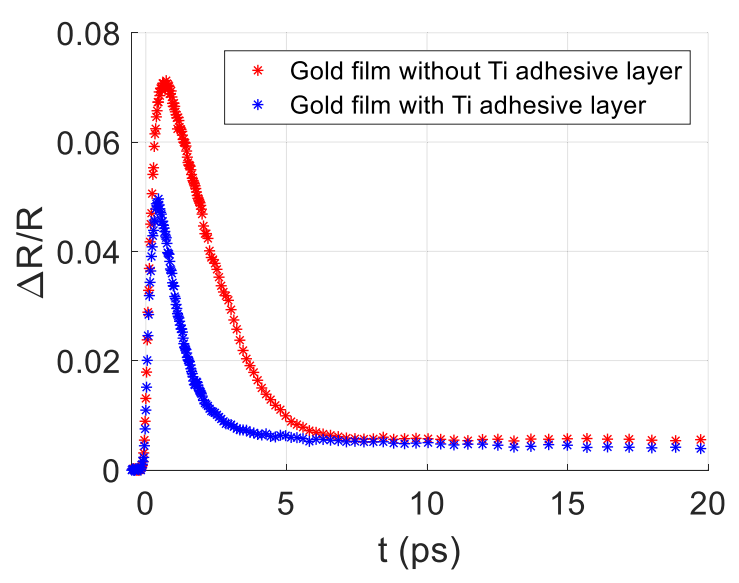

FIG. 8. Experimental profiles of transient reflectivity for a 50-nm- gold film with (blue dots) and without (red dots) titanium adhesion layer.

could also contribute to its amplitude. Both positions of the $\Delta T / T$ maxima are close but not equal to their corresponding absorption spectral resonances. Also, the signal magnitude is almost three times higher for the parallel component than for the perpendicular component, this discrepancy being directly linked to the difference in resonances amplitude along the two directions. As for thin films, the large variation of the electronic temperature during the first picosecond, changes the interband transition through a modification of the Fermi distribution and the intraband transition through the modification of the electron scattering time in the Drude model. The results of these two contributions is an overall shift and attenuation of the nanorods resonances that can be seen in the graphs of Fig. 9.

Finally, Fig. 10 shows the time-resolved relative transmission measured at the resonance of the structures, for the two polarization configurations and for five different pump fluences. The model predictions are shown in solid lines, with the same parameters used for all fluences. These parameters are identical for both polarizations. For these nanorods, variation of permittivity with lattice temperature, using the Drude model previously described in Eq. (10), gives $\delta \varepsilon / \delta T=0.8 \times 10^{-3}+i 2.4 \times 10^{-3} \mathrm{~K}^{-1}$ at $523 \mathrm{~nm}$ and $\delta \varepsilon / \delta T=0.4 \times 10^{-3}+i 1.3 \times 10^{-3} \mathrm{~K}^{-1}$ at $618 \mathrm{~nm}$. The best fitting curves were obtained for the following parameters: $G_{0}=0.68[0.61 ; 0.74] \times 10^{16} \mathrm{~W} \mathrm{~m}^{-3}$, $d G / d T_{e}=0[0 ; 0.005] \times 10^{13} \mathrm{~W} \mathrm{~m}^{-3} \mathrm{~K}^{-1}$ and $C=$ $1.63[1.60 ; 1.66] \times 10^{-9} \mathrm{eVm}$. In particular, the lower energy coupling between electrons and phonons could be explained by a lower carrier density for nanorods in comparison with continuous gold films [41,42]. Also, to consider the difference in plasmonic resonances for nanorods compared to flat gold films, we use the energy gap between the $\mathrm{d}$ band and Fermi level as a fit parameter for which we find $E_{g}=2.35 \mathrm{eV}(2.54 \mathrm{eV}$ for

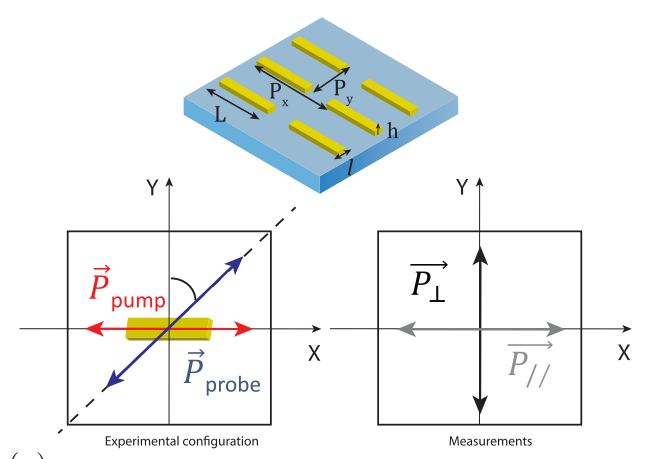

(a)

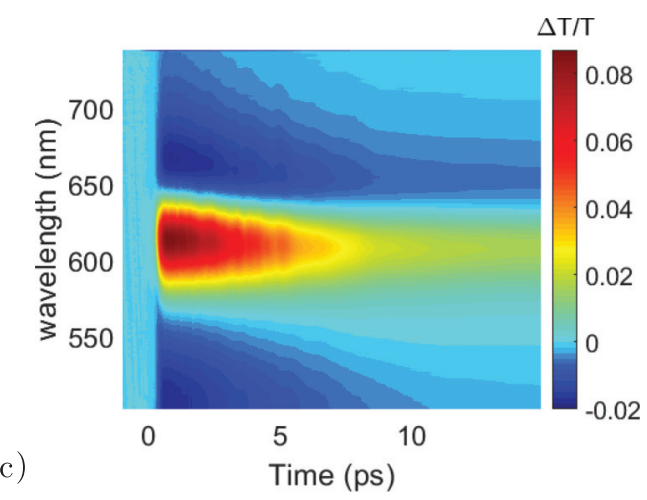

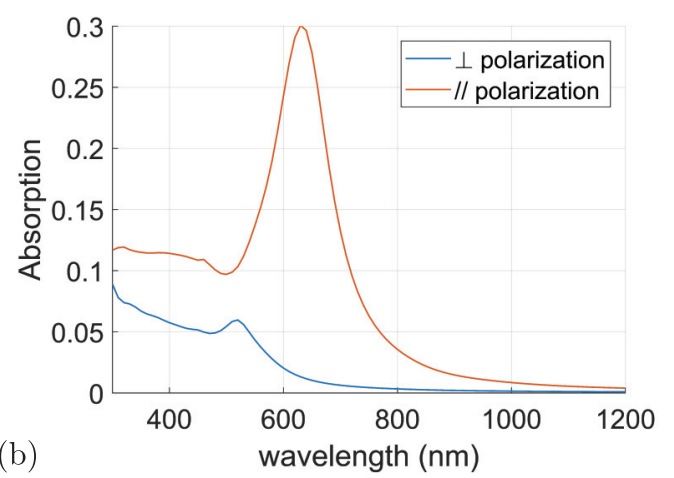

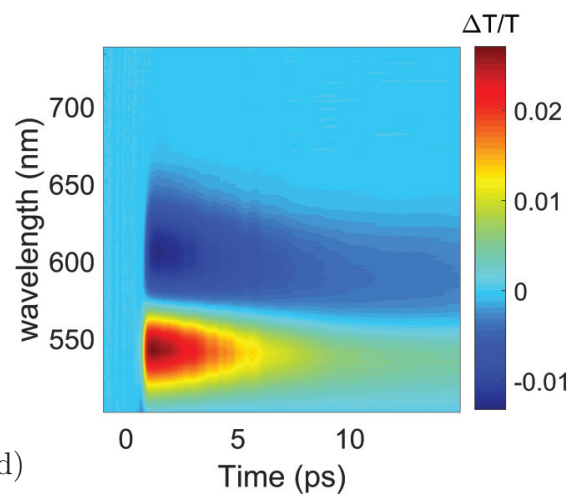

FIG. 9. (a) Schematic of the nanostructured sample and polarization configuration; (b) absorption spectrum for the two different incidences of polarization (perpendicular and parallel to the rod axis); (c) Spectrotemporal transmission map for the parallel polarization component of the probe beam; (d) Spectrotemporal transmission map for the perpendicular polarization component of the probe beam. 

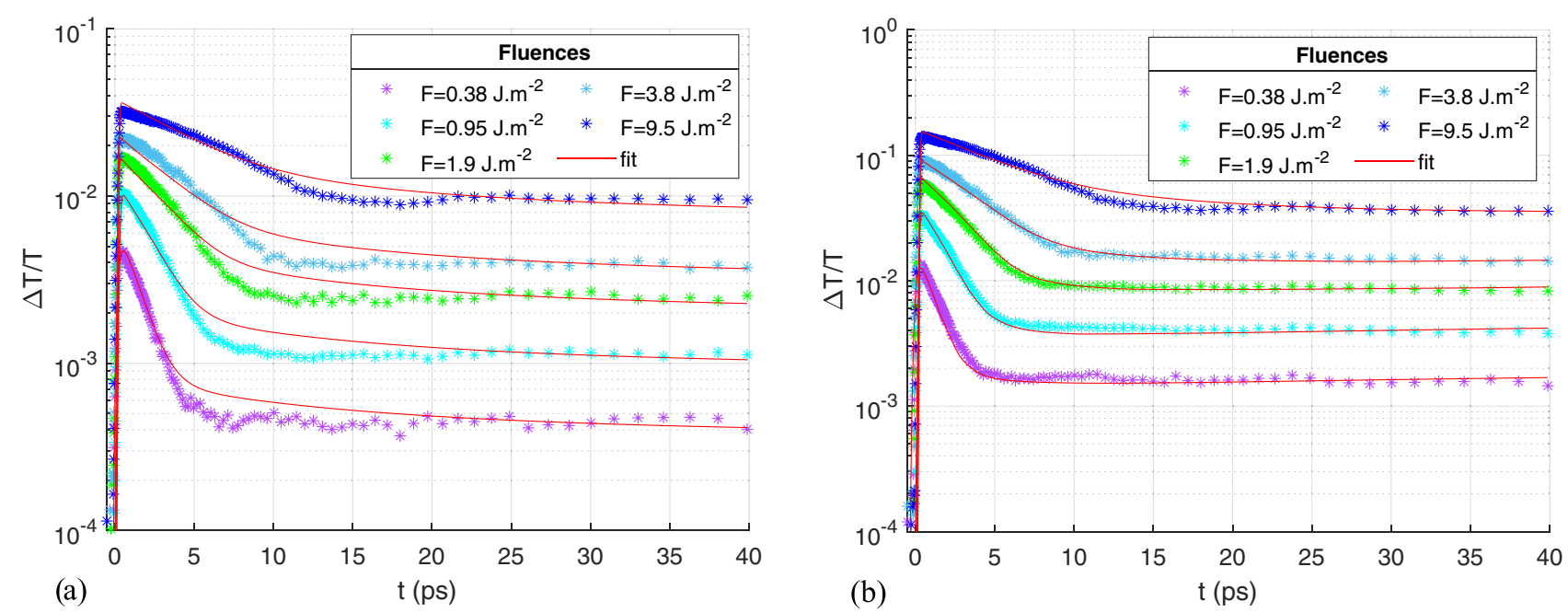

FIG. 10. Best fit of the maximum of variation of transmission as a function of time, for an array of gold nanorods (40 x $80 \mathrm{x} 30 \mathrm{~nm})$ on glass substrate with a pump at $\lambda=400 \mathrm{~nm}$ and at different pump powers when: (a) probe beam is polarized along the width of nanorods, and (b) probe beam is polarized along the length of the nanorods.

flat gold). This led to a better estimation of the interband contribution to the gold permittivity evolution with electronic temperature.

\section{CONCLUSION}

In conclusion, we have developed and experimentally verified an improved numerical model of fast thermoplasmonics phenomena. This model combines a modified two-temperature model, with the Rouard method for thin films and a hybrid approach combining finite elements with the Fourier modal method for nanorod arrays. Using a pumpprobe setup, we acquired spectrotemporal transient reflectivity maps for thin gold films of different thicknesses under a wide range of pump fluences. The generalized numerical model is integrated in a fitting algorithm that allows us to reproduce the entire set of experimental data. The agreement between the numerical results and the experimental curves is remarkably good over a wide range of pump-probe delays, considering the small number of free parameters of the model. The analysis shows that the temperature dependence of gold permittivity plays a major role on the dynamics of the thermoplasmonics phenomenon. We also demonstrated the significant impact of the thin titanium adhesion layer underlying the gold films.
Importantly, the model also fit the transient transmission response of array of gold nanorods on glass substrate equally well. As this model enables the accurate determination of the spatial and temporal distribution of temperature inside the sample from the pump-probe optical data, it could be used to predict and optimize thermoplasmonics effects for any geometry of nanostructures. Further experiments may use the model to explore various materials with appropriate optical values.

\section{ACKNOWLEDGMENTS}

This work was supported by the Natural Sciences and Engineering Research Council of Canada (NSERC) with Discovery Grants for M.C. and P.G.C. P.B. is supported by a doctoral scholarship from a French doctoral contract (MESRI) with the Université Paris-Saclay. J-F.B. is supported by the Fonds de recherche du Québec-Nature et technologies (FRQNT) and his Merit scholarship program for foreign students (No. 273433). LN2 is an international laboratory (Unité Mixte Internationale UMI 3463) jointly managed by French CNRS and the Université de Sherbrooke as well as Université de Lyon (ECL, INSA de Lyon, CPE) and the Université Grenoble-Alpes (UGA). LN2 is also financially supported by FRQNT.
[1] A. O. Govorov and H. H. Richardson, Nano Today 2, 30 (2007).

[2] G. Baffou, C. Girard, and R. Quidant, Phys. Rev. Lett. 104, 136805 (2010).

[3] P. Ben-Abdallah, R. Messina, S.-A. Biehs, M. Tschikin, K. Joulain, and C. Henkel, Phys. Rev. Lett. 111, 174301 (2013).

[4] G. Han, P. Ghosh, M. De, and V. M. Rotello, NanoBiotechnol. 3, 40 (2007).

[5] B. C. Stipe, T. C. Strand, C. C. Poon, H. Balamane, T. D. Boone, J. A. Katine, J.-L. Li, V. Rawat, H. Nemoto, A. Hirotsune, O. Hellwig, R. Ruiz, E. Dobisz, D. S. Kercher, N. Robertson, T. R. Albrecht, and B. D. Terris, Nat. Photon. 4, 484 (2010).

[6] P. Christopher, H. Xin, and S. Linic, Nat. Chem. 3, 467 (2011).
[7] N. Li, J. Ren, L. Wang, G. Zhang, P. Hänggi, and B. Li, Rev. Mod. Phys. 84, 1045 (2012).

[8] P. Ben-Abdallah, Phys. Rev. Lett. 116, 084301 (2016).

[9] R. S. DiMatteo, P. Greiff, S. L. Finberg, K. A. Young-Waithe, H. K. H. Choy, M. M. Masaki, and C. G. Fonstad, Appl. Phys. Lett. 79, 1894 (2001).

[10] S. I. Anisimov, B. L. Kapeliovich, and T. L. Perel'man, J. Exp. Theor. Phys. 39, 375 (1974).

[11] T. J. Derrien, T. Sarnet, M. Sentis, and T. E. Itina, J. Optoelectron. Adv. Mater. 12, 610 (2010).

[12] T. Shin, S. W. Teitelbaum, J. Wolfson, M. Kandyla, and K. A. Nelson, J. Chem. Phys. 143, 194705 (2015).

[13] L. Jiang and H.-L. Tsai, J. Heat Transfer 127, 1167 (2005). 
[14] T. Labouret and B. Palpant, Phys. Rev. B 94, 245426 (2016).

[15] E. Majchrzak and J. Dziatkiewicz, Science 11, 63 (2012).

[16] M. Ortolani, A. Mancini, A. Budweg, D. Garoli, D. Brida, and F. de Angelis, Phys. Rev. B 99, 035435 (2019).

[17] N. Singh, Int. J. Mod. Phys. B 24, 1141 (2010).

[18] E. Carpene, Phys. Rev. B 74, 024301 (2006).

[19] C.-K. Sun, F. Vallée, L. H. Acioli, E. P. Ippen, and J. G. Fujimoto, Phys. Rev. B 50, 15337 (1994).

[20] P. B. Allen, Phys. Rev. Lett. 59, 1460 (1987).

[21] W. S. Fann, R. Storz, H. W. K. Tom, and J. Bokor, Phys. Rev. Lett. 68, 2834 (1992).

[22] B. Rethfeld, A. Kaiser, M. Vicanek, and G. Simon, Phys. Rev. B 65, 214303 (2002).

[23] G. Della Valle, M. Conforti, S. Longhi, G. Cerullo, and D. Brida, Phys. Rev. B 86, 155139 (2012).

[24] P. Lecaruyer, E. Maillart, M. Canva, and J. Rolland, Appl. Opt. 45, 8419 (2006).

[25] J. P. Hugonin, M. Besbes, and P. Lalanne, Opt. Lett. 33, 1590 (2008).

[26] P. E. Hopkins and P. M. Norris, J. Heat Trans. 131, 043208 (2009).

[27] A. Suslova and A. Hassanein, J. Appl. Phys. 124, 065108 (2018).

[28] X. Y. Wang, D. M. Riffe, Y.-S. Lee, and M. C. Downer, Phys. Rev. B 50, 8016 (1994).

[29] X. Wang, K. Chen, M. Zhao, and D. D. Nolte, Opt. Express 18, 24859 (2010).

[30] R. Rosei and D. W. Lynch, Phys. Rev. B 5, 3883 (1972).

[31] R. Rosei, F. Antonangeli, and U. M. Grassano, Surf. Sci. 37, 689 (1973).
[32] R. Rosei, C. H. Culp, and J. H. Weaver, Phys. Rev. B 10, 484 (1974).

[33] R. Rosei, Phys. Rev. B 10, 474 (1974).

[34] N. E. Christensen and B. O. Seraphin, Phys. Rev. B 4, 3321 (1971).

[35] F. Wooten, Optical Properties of Solids (Academic Press, New York, 1972).

[36] T. Srivastava, R. Das, and R. Jha, Plasmonics 8, 515 (2013).

[37] O. A. Yeshchenko, I. S. Bondarchuk, V. S. Gurin, I. M. Dmitruk, and A. V. Kotko, Surf. Sci. 608, 275 (2013).

[38] H. Reddy, U. Guler, A. V. Kildishev, A. Boltasseva, and V. M. Shalaev, Opt. Mater. Express 6, 2776 (2016).

[39] Z. Lin, L. V. Zhigilei, and V. Celli, Phys. Rev. B 77, 075133 (2008).

[40] V. G. Kravets, F. Schedin, and A. N. Grigorenko, Nat. Commun. 3, 640 (2012).

[41] R. Dick, Nanoscale Res. Lett. 5, 1546 (2010).

[42] G. Bisker and D. Yelin, J. Opt. Soc. Am. B 29, 1383 (2012).

[43] See Supplemental Material at http://link.aps.org/supplemental/ 10.1103/PhysRevB.102.155127 for (1) the impact on the calculated relative reflectivity of a $+10 \%$ variation on the thermal and optical parameters used in the model in order to identify the most relevant parameters; (2) a comparison of heat transfer efficiencies for the different mechanisms: conduction, convection and black body emission; (3) the gold band diagram with the relevant interband transitions; (4) the electronic and lattice temperature spatial distribution maps inside a thin gold film (thickness of $50 \mathrm{~nm}$ ); (5) the permittivity of gold as a function of temperature used in our model, measured by ellipsometry; (6) the calculation of the pump and probe overlap to find the energy distribution on the sample. 